\title{
OPEN Functional buffering via cell-specific gene expression promotes tissue homeostasis and cancer robustness
}

\author{
Hao-Kuen Lin ${ }^{1,2,4}$, Jen-Hao Cheng ${ }^{1,3,4}$, Chia-Chou Wu ${ }^{1,4}$, Feng-Shu Hsieh ${ }^{1}$, Carolyn Dunlap ${ }^{1}$ \& \\ Sheng-hong Chen ${ }^{1,3 凶}$
}

Functional buffering that ensures biological robustness is critical for maintaining tissue homeostasis, organismal survival, and evolution of novelty. However, the mechanism underlying functional buffering, particularly in multicellular organisms, remains largely elusive. Here, we proposed that functional buffering can be mediated via expression of buffering genes in specific cells and tissues, by which we named Cell-specific Expression-BUffering (CEBU). We developed an inference index (C-score) for CEBU by computing C-scores across 684 human cell lines using genome-wide CRISPR screens and transcriptomic RNA-seq. We report that C-score-identified putative buffering gene pairs are enriched for members of the same duplicated gene family, pathway, and protein complex. Furthermore, CEBU is especially prevalent in tissues of low regenerative capacity (e.g., bone and neuronal tissues) and is weakest in highly regenerative blood cells, linking functional buffering to tissue regeneration. Clinically, the buffering capacity enabled by CEBU can help predict patient survival for multiple cancers. Our results suggest $C E B U$ as a potential buffering mechanism contributing to tissue homeostasis and cancer robustness in humans.

Robustness in biological systems is critical for organisms to carry out vital functions in the face of environmental challenges ${ }^{1,2}$. A fundamental requirement for achieving biological robustness is functional buffering, whereby the biological functions performed by one gene can also be attained via other buffering genes. Although functional buffering has long been regarded as a critical function contributing to biological robustness, the mechanisms underlying functional buffering remain largely unclear ${ }^{3}$. Based on transcriptional regulation of buffering genes, functional buffering can be categorized as either needs-based buffering or intrinsic buffering. Needs-based buffering involves transcriptional activation of buffering genes only when the function of a buffered gene is compromised. To accomplish needs-based buffering, a control system must exist to detect compromised function and then activates expression of buffering genes. Needs-based buffering is often observed as genetic compensation in various biological systems including fungi, animals and plants ${ }^{3-6}$. One classical needs-based buffering mechanism is genetic compensation among duplicated genes, whereby expression of a paralogous gene is upregulated when the function of the active duplicated gene is compromised ${ }^{4}$. Genetic analyses of duplicated genes in Saccharomyces cerevisiae have revealed upregulation of gene expression in $\sim 10 \%$ of paralogs when cell growth is compromised due to deletions of their duplicated genes ${ }^{6-8}$. Apart from duplicated genes, non-orthologous/analogous genes can also be activated for needs-based buffering 9 . For instance, inactivation of one growth signaling pathway can lead to activation of others for the coordination of cell growth and survival ${ }^{3,4}$. Such needs-based buffering genes have been documented as enabling unicellular/multicellular organisms to cope with environmental stresses ${ }^{3,8}$.

Recent genome-wide studies of duplicated genes in human cells have revealed another class of buffering mechanism whereby expression of buffering genes is not responsive to impaired function but is constitutively expressed, hereafter termed "intrinsic buffering" ${ }^{10-12}$. In some duplicated gene families, the strength of paralogous gene expression determines the essentiality of their corresponding duplicated genes in human cell lines, i.e., the higher the expression of paralogous genes in a particular cell line, the less essential are their duplicated genes ${ }^{10-12}$. This observation indicates that paralogs may buffer and contribute to the function of their duplicated genes in specific cells through their constitutive gene expression. In addition to duplicated gene families, gene essentiality can depend on inherent variability in the expression levels of other genes in the same pathway, suggesting that functionally analogous genes in the same pathway can also buffer each other ${ }^{13}$. Despite these observations, it

\footnotetext{
${ }^{1}$ Lab for Cell Dynamics, Institute of Molecular Biology, Academia Sinica, Taipei 115, Taiwan. ${ }^{2}$ College of Medicine, National Taiwan University, Taipei 106, Taiwan. ${ }^{3}$ Genome and Systems Biology Degree Program, Academia Sinica and National Taiwan University, Taipei, Taiwan. ${ }^{4}$ These authors contributed equally: Hao-Kuen Lin, Jen-Hao Cheng and Chia-Chou Wu. ${ }^{\square}$ email: shengchen@gate.sinica.edu.tw
} 
A

\section{Cell-specific Expression-BUffering mechanism (CEBU)}
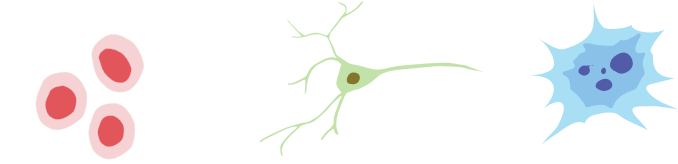

Buffering Gene Expression
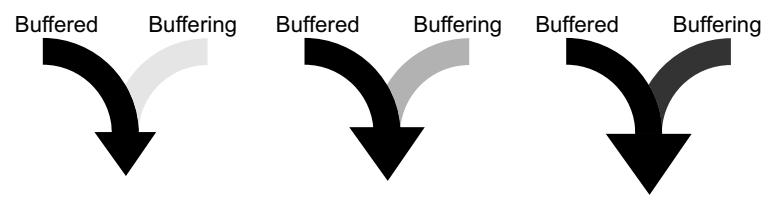

Buffering Capacity

Buffered Gene Essentiality
B

\section{C-score plot}

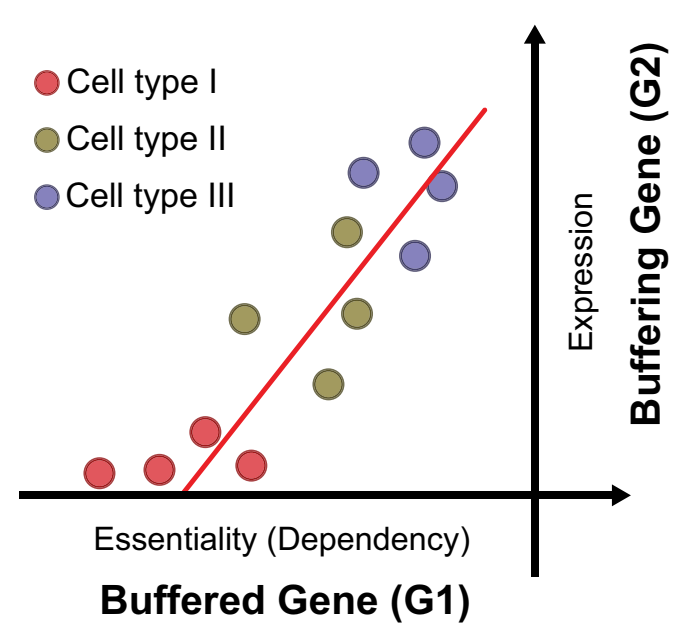

C

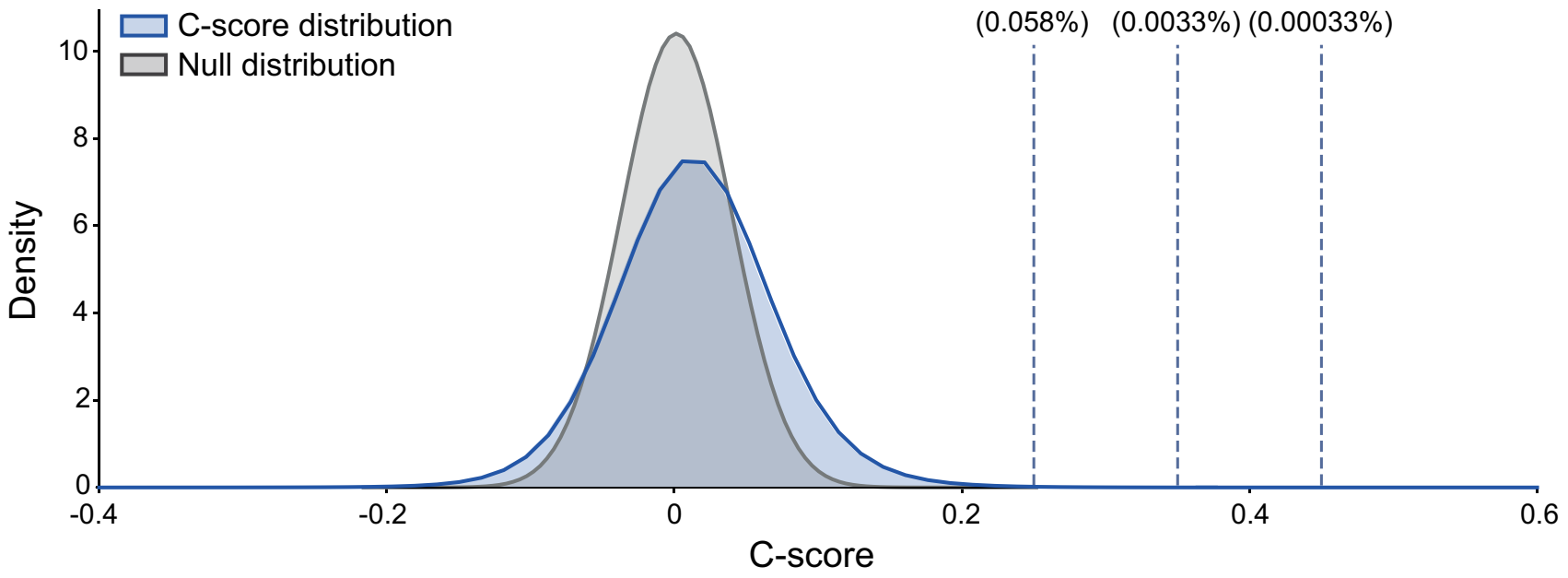

Figure 1. Genome-wide CEBU analysis using the C-score index. (A) Illustration of the cell-specific expressionbuffering mechanism (CEBU). Cells with higher buffering gene (G2) expression (darker arrow) have stronger buffering potential, thereby lowering the essentiality of the buffered gene (G1). The illustration and cartoons are created using Adobe Illustrator. (B) C-score plot: the $\mathrm{x}$-axis is the dependency score of the buffered gene (G1) and the $y$-axis is the expression level of the buffering gene (G2). G1 is considered as being potentially buffered by $\mathrm{G} 2$, as quantified by $\mathrm{C}$-score, which is an adjusted correlation for a given gene based on the C-score plot. (C) Distribution of C-scores for all gene pairs (blue) and one of five randomly shuffled distributions (grey). The plot shows C-score between 0.4 and 0.6 . The dashed lines indicate the $\mathrm{C}$-score percentiles of $0.25,0.35$ and 0.45 for the all-gene-pair distribution.

remains unclear what mechanism may give rise to this context-dependent constitutive expression of buffering genes and how such intrinsic buffering may function in multicellular organisms.

In this study, we investigated if cell- and tissue-specific gene expression can act as an intrinsic buffering mechanism to buffer functionally related genes in the genome, thereby strengthening cellular plasticity for cell- and tissue-specific tasks. We proposed this concept as the "Cell-specific Expression-BUffering" or CEBU mechanism (illustrated in Fig. 1A). To explore CEBU as a potential buffering mechanism and to estimate buffering capability, we developed an inference index, the C-score, to identify putative gene pairs displaying CEBU. This index calculates the adjusted correlation between expression of a buffering gene and the essentiality of the buffered gene (Fig. 1B), utilizing transcriptomics data ${ }^{14}$ and genome-wide dependency data from the DepMap project $^{14,15}$ across 684 human cell lines. Our results suggest the potential authenticity of the CEBU mechanism 


\begin{tabular}{|l|l|l|l|l|}
\hline & G1 & G2 & C-score & Duplicated or non-duplicated \\
\hline 1 & FAM50A & FAM50B & 0.793 & $\mathrm{D}$ \\
\hline 2 & RPP25L & RPP25 & 0.770 & $\mathrm{D}$ \\
\hline 3 & CDS2 & CDS1 & 0.677 & $\mathrm{D}$ \\
\hline 4 & EFR3A & EFR3B & 0.657 & $\mathrm{D}$ \\
\hline 5 & RAB6A & RAB6B & 0.639 & $\mathrm{D}$ \\
\hline 6 & INTS6 & INTS6L & 0.624 & $\mathrm{D}$ \\
\hline 7 & CHMP4B & CHMP4C & 0.618 & $\mathrm{D}$ \\
\hline 8 & ATP6VOE1 & ATP6V0E2 & 0.610 & $\mathrm{D}$ \\
\hline 9 & TTC7A & TTC7B & 0.609 & $\mathrm{D}$ \\
\hline 10 & DNAJC19 & DNAJC15 & 0.606 & $\mathrm{D}$ \\
\hline 11 & STX4 & STX2 & 0.600 & $\mathrm{D}$ \\
\hline 12 & IRF4 & PTK2 & 0.588 & $\mathrm{ND}$ \\
\hline 13 & PCYT1A & PCYT1B & 0.578 & $\mathrm{D}$ \\
\hline 14 & NMT1 & NMT2 & 0.562 & $\mathrm{D}$ \\
\hline 15 & SNAP23 & SNAP25 & 0.552 & $\mathrm{D}$ \\
\hline 16 & ATP1B3 & ATP1B1 & 0.547 & $\mathrm{D}$ \\
\hline 17 & MYB & CAMSAP2 & 0.547 & $\mathrm{ND}$ \\
\hline 18 & MYBL2 & MYBL1 & 0.544 & $\mathrm{D}$ \\
\hline 19 & TP53BP1 & EDA2R & 0.540 & $\mathrm{ND}$ \\
\hline 20 & POP7 & RPP25 & 0.537 & ND \\
\hline
\end{tabular}

Table 1. Top $20 \mathrm{C}$-score gene pairs. The top $20 \mathrm{G} 1$ s pairing with the $\mathrm{G} 2$ that yields the highest C-score is shown. $D$ duplicated gene pair, ND Non-duplicated gene pair.

and this CEBU-mediated intrinsic buffering may play a critical role in cell-specific survival, tissue homeostasis, and cancer robustness.

\section{Results}

Development of the C-score to infer cell-specific expression buffering (CEBU). In seeking an index to explore intrinsic buffering operated via constitutive gene expression, we postulated a buffering relationship whereby the essentiality of a buffered gene (G1) increases when expression of its buffering gene (G2) decreases across different human cell lines (Fig. 1). Given that G2 expression differs among cell lines, the strength of buffering capacity varies across cell lines, thereby conferring on G1 cell-specific essentiality. This concept prompted the formulation of the cell-specific expression buffering mechanism, here named CEBU, which can be quantitatively investigated by developing an inference index, C-score. The $\mathrm{C}$-score of a gene pair is derived from the correlation between the essentiality of a buffered gene (G1) and expression of its buffering gene (G2) (see C-score plot, Fig. 1B), and is formulated as:

$$
\text { C-score }=\rho_{G 1, G 2}\left(1+b \frac{\text { slope }_{\text {min }}}{\text { slope }_{G 1, G 2}}\right)
$$

where $\rho$ denotes the Pearson correlation coefficient between essentiality of G1 and expression of G2. Their regression slope $\left(s l o p e_{G 1, G 2}\right)$ is normalized by slope $e_{m i n}$, which denotes the minimum slope of all considered gene pairs in the human genome (see "Methods"). The normalized slope can be weighted by cell- or tissue type-specific $b$. In our current analysis, $b$ is set as 1 for a pan-cell- and pan-cancer-type analysis. Gene essentiality is represented by dependency scores (D.S.) from the DepMap project ${ }^{15}$, where the effect of each gene on cell proliferation was quantified after its knockout using the CRISPR/Cas-9 approach. Specifically, a more negative D.S. reflects slower cell proliferation when the gene is knocked out, thus reflecting stronger essentiality. Expression data was obtained through RNA-seq ${ }^{14}$. We anticipated that the higher the C-score of a gene pair, the more likely G2 would buffer G1 based on our proposed CEBU mechanism.

We conducted a genome-wide analysis to calculate C-scores for all genes with negative mean dependency scores (G1s) pairing with expressed genes (G2s), yielding $9196 \mathrm{G} 1 \times 13,577 \mathrm{G} 2$ gene pairs across 684 human cell lines (Fig. 1C and see "Methods"). For our analysis, we considered gene pairs to have a high C-score with a strong likelihood of intrinsic buffering when their C-scores were $>0.25$, yielding 64,439 gene pairs which comprises $0.058 \%$ of the $9196 \times 13,577$ gene pairs in the human genome (Supplementary Table S1 and Data availability). To assess whether the results are due to random chance, we generated a bootstrapped null distribution by random shuffling of G2 expression among cell lines (Fig. 1C). This null distribution can be modeled as a normal distribution (Supplementary Fig. S1), for which we determined the high C-score gene pairs are statistically significant with a $q$-value $<2.2 \mathrm{e}-16$ after multiple testing correction (Fig. 1C). Table 1 lists the top $20 \mathrm{G} 1 \mathrm{~s}$ pairing with the $\mathrm{G} 2$ that yields the highest $\mathrm{C}$-score. The highest C-score gene pair is FAM50A and FAM50B, which are potential transcriptional regulators recently discovered to be synthetic lethal ${ }^{16}$. Other well described synthetic lethal cases 
include $R P P 25 L / R P P 25^{16-18}, C D S 2 / C D S 1^{16}$ (Table 1) as well as other high C-score gene pairs, UAP1/UAP1L1 ${ }^{16,19,20}$, SMARC4/SMARC2 $2^{16,18,20,21}$, and ENO1/ENO2 ${ }^{22}$ (Supplementary Table S1). Comparing with nine other predictions of synthetic lethality ${ }^{23-31}$ to four larger scale studies of experimental evidences ${ }^{16,19,20,32}$, the high C-score gene pairs demonstrated among the highest number of matches to experiments, even though matches between prediction to experiments remains few (0 to $14.3 \%$, Supplementary Table S2). This result indicate identification of buffering and synthetic lethality is non-trivial, possibly due to a wide variety of biological mechanisms, and the CEBU concept may be one of these mechanisms. Furthermore, based on the concept of CEBU, a high C-score should be indicative of marked variability in cell-specific essentiality and expression. Indeed, we observed higher variability in both G1 dependency and G2 expression for high C-score gene pairs (Supplementary Fig. S2A). Nevertheless, high variation alone is insufficient to grant a high $\mathrm{C}$-score. A high C-score requires consistent pairing between G1 and G2 across cell lines and, as anticipated, disrupting the pairing between G1 dependency and G2 expression by shuffling G2 expression amongst cell lines (without changing variability) abolished the C-score relationship (compare the right and left panels of Supplementary Fig. S2B). Moreover, both mean G1 dependency and mean G2 expression in high C-score gene pairs were lower than those parameters in randomly selected gene pairs (Supplementary Fig. S2C), implying that G1s and G2s in high C-score gene pairs tend to be more essential and less expressed, respectively.

The proposed CEBU mechanism describes an intrinsic buffering mechanism that functions under normal physiological conditions. Consistent with this notion, when we examined if the C-score index could be biased due to the usage of cancer cell lines, we found that only a low percentage (2.3\% per gene pair, Supplementary Fig. S3A) of mutant cell lines contributed to our C-score measurements. Moreover, excluding mutant cell lines did not qualitatively affect our C-score measurements, especially for high C-score gene pairs (Supplementary Fig. S3B). The same trend holds for cancer-related genes (Supplementary Fig. S3B). These results indicate that mutant cell lines are not the major determinants of C-scores. Similarly, since copy number variation (CNV) is a major mechanism for oncogenic expression, we checked if CNV contributes to G2 expression. As shown in Supplementary Fig. S3C, the correlation between G2 expression and copy number decreases with increasing $\mathrm{C}$-score, indicating that $\mathrm{CNV}$ is not a primary mechanism regulating $\mathrm{G} 2$ expression. Thus, the C-score index is likely not biased by the utilization of cancer cell lines. Moreover, genes located chromosomal adjacently in the same topological associating domain (TAD) tend to co-express ${ }^{33}$. Hence, we interrogated if G2 is adjacent to a duplicated gene of G1. We found only 15,582/64,439 (24.2\%) G2s are on the same chromosome as any G1 duplicated gene. Within these, the minimum distances between a G2 to any G1 duplicated gene are generally greater than the typical size of a TAD $\left(750 \mathrm{kbp}^{34}\right.$ to $\left.2.5 \mathrm{Mbps}^{35}\right)$, indicating that $\mathrm{G} 2$ is not selected by adjacency to G1 duplicated gene (Supplementary Fig. S3D).

Characterization of C-score-inferred CEBU gene pairs. Since several duplicated gene pairs have been implicated as displaying functional buffering via gene expression ${ }^{10-12}$, we checked if C-score identified duplicated gene pairs are over-represented. Among the high C-score gene pairs, 210 pairs are duplicated gene pairs. We found that duplicated gene pairs are more enriched among high C-score gene pairs as C-score increases (Fig. 2A and see "Methods"), where statistically significant enrichment is reached at C-scores $>0.255$ ( $p$-value $=0.05$ by using a hypergeometric test). This enrichment increases with higher C-scores suggests a higher likelihood that CEBU describes a potential buffering mechanism utilize by a portion of duplicated genes (Fig. 2A). Interestingly, the majority of high C-score gene pairs are non-duplicated ( $>90 \%$, Fig. $2 \mathrm{~B})$. In these cases, we asked if G2s may be functional analogs of the respective G1s, acting as surrogate genes. Accordingly, we examined if the high C-score gene pairs are more likely to participate in the same function or biological pathway or physically interact. We calculated the enrichment of curated gene sets in terms of Gene Ontology (GO) ${ }^{36}$ and Kyoto Encyclopedia of Genes and Genomes (KEGG) ${ }^{37}$ from the Molecular Signatures Database ${ }^{38}$ (Fig. 2C). Gene pairs with high C-scores consistently exhibited greater functional enrichment. Likewise, we observed a monotonic increase in the enrichment of protein-protein interactions (PPI) [using the STRING ${ }^{39}$ and CORUM $^{40}$ databases] between G1s and G2s in accordance with increasing C-score cutoff (Fig. 2D). To gain insight into which functions are represented in the proposed CEBU mechanism, we considered that G1 may be paired with multiple G2s and vice versa, and constructed a C-score network consisting of G1s, G2s, and genes that act as both G1s and G2s (Fig. 2E). The enriched functions include housekeeping functions such as regulating redox homeostasis, gene transcription, mRNA translation, as well as NTP synthesis (Fig. 2E). Moreover, some cancer-related pathways are also enriched in the C-score-identified buffering network, including the proto-oncogenes EGFR and MYC (Fig. 2E). Both duplicated and non-duplicated gene pairs contribute to the observed functional and PPI enrichments. However, notably, the increase in functional and PPI enrichment as C-score increases are primarily attributable to non-duplicated genes as there is an observable increase in enrichment for all genes while enrichment for duplicated genes is relatively constant with increasing C-scores (compare Fig. 2C,D to Supplementary Fig. S4A,B). These results indicate a strong likelihood for intrinsic buffering among analogous genes in the same pathway or proteins in the same protein complex. Thus, high C-score gene pairs are enriched in duplicated gene pairs, as well as non-duplicated gene pairs that are members of the same biological pathway and/or encode physically interacting proteins, supporting that the proposed CEBU mechanism (which is the basis of the C-score index) may provide an explanation to a part of intrinsic buffering between such gene pairs.

Experimental validation of C-score-inferred CEBU gene pairs. To validate putative C-score-inferred buffering gene pairs, we conducted experiments on the highest C-score gene pair, i.e., FAM50A-FAM50B, both members of which belong to the same duplicated gene family. Based on a C-score plot of FAM50A-FAM50B (Fig. 3A), we expected that $F A M 50 B$ would display a stronger buffering effect on FAM50A for cell lines located at the top-right of the plot (e.g. MCF7) relative to those at the bottom-left (e.g. U2OS). Accordingly, growth of 
A

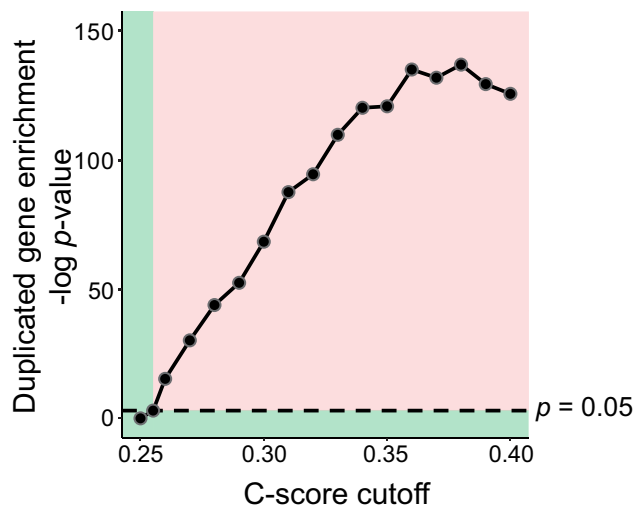

C

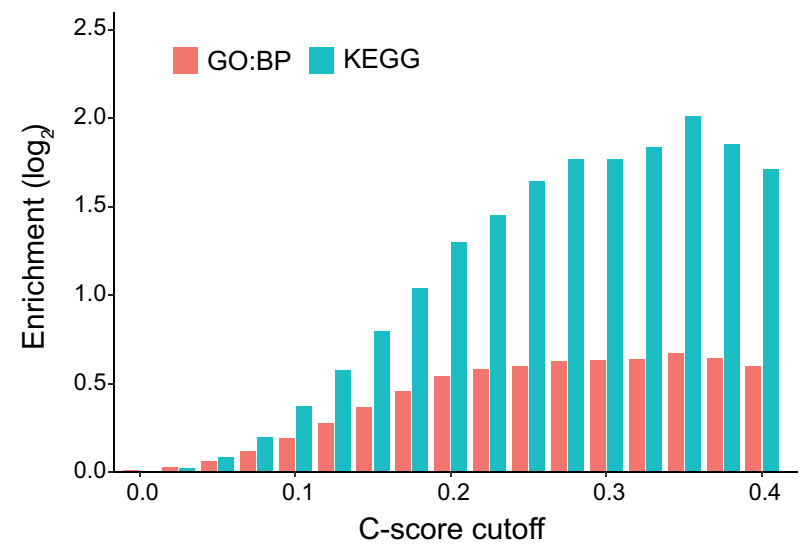

B

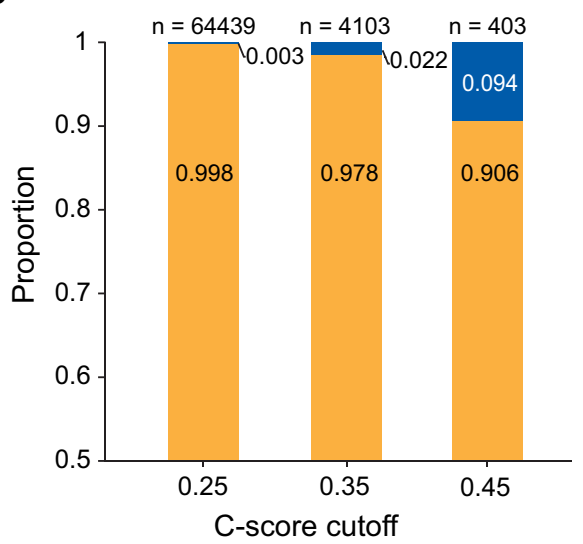

D
- Duplicated genes

Non-duplicated genes
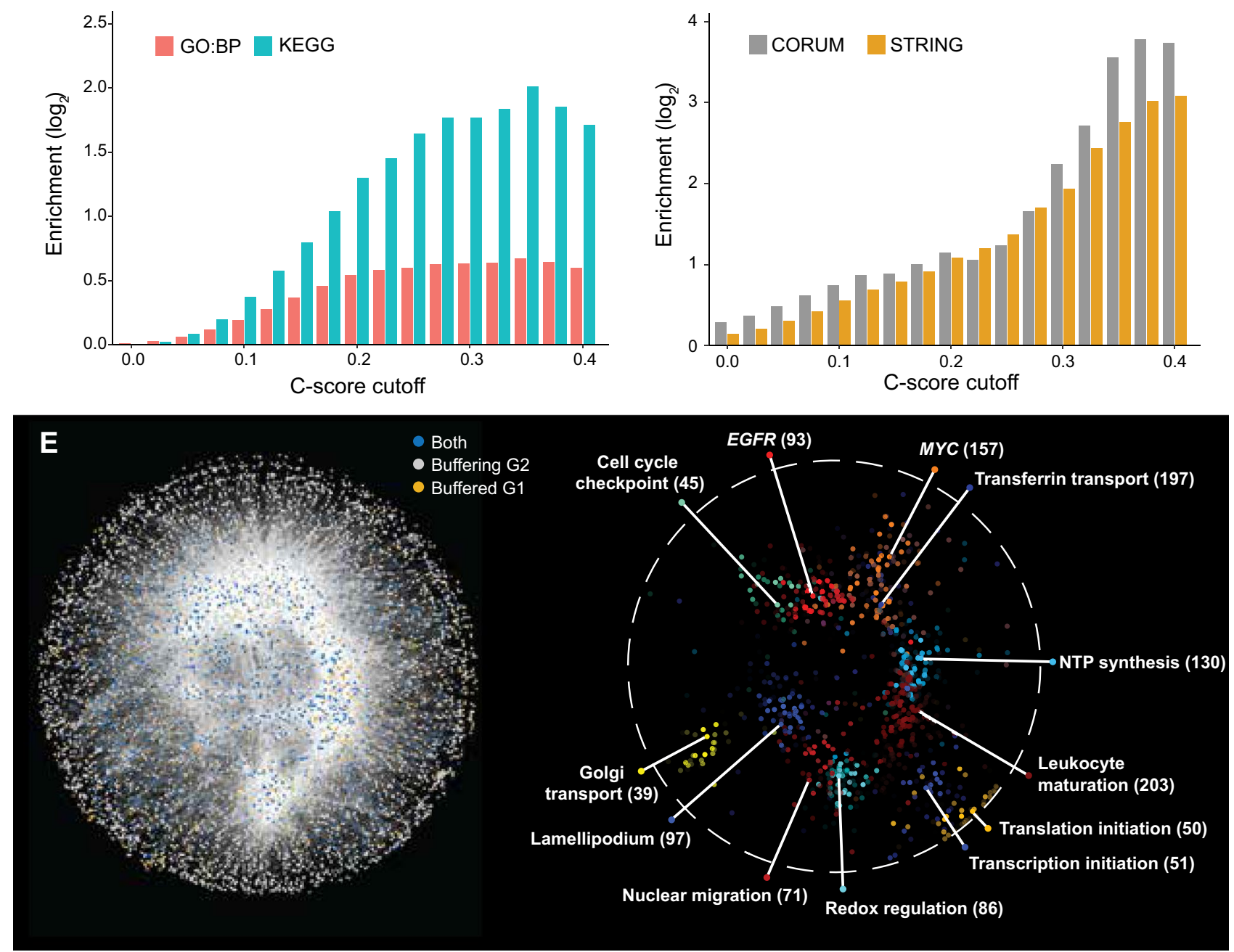

Figure 2. Properties of high C-score gene pairs. (A) Enrichment for duplicated genes as C-scores increase (hypergeometric test). The dashed line denotes $p$-value of 0.05 , where the corresponding C-score is 0.255 . The red region (i.e., above the dashed line and equating to $\mathrm{C}$-score $>0.255$ ) indicates significant enrichment. The green region indicates lack of significance. (B,C) Functional enrichment of C-score gene pairs increases with C-score cutoff. (B) Percentage of C-score-identified non-duplicated and duplicated gene pairs with different C-score cut-offs. (C) Enrichment of pairs of genes annotated with the same gene ontology biological process (GO:BP) term or KEGG pathway in C-score gene pairs. Enrichment increases with C-score cut-off. (D) Enrichment of pairs of genes with annotated protein-protein interactions from STRING and within the same protein complex from CORUM among C-score gene pairs. Enrichment increases with C-score cut-off. (E) Left: the buffering gene network is composed of 6664 nodes and 42,754 edges with C-scores $>0.2536$ visualized using Cytoscape (https://cytoscape.org/). Orange nodes represent buffered genes; grey nodes are buffering genes, and blue nodes are genes that are both buffered and buffering. Right: Clusters of GO-enriched biological functions in the buffering gene network. Numbers indicate the number of genes in the cluster. 
A

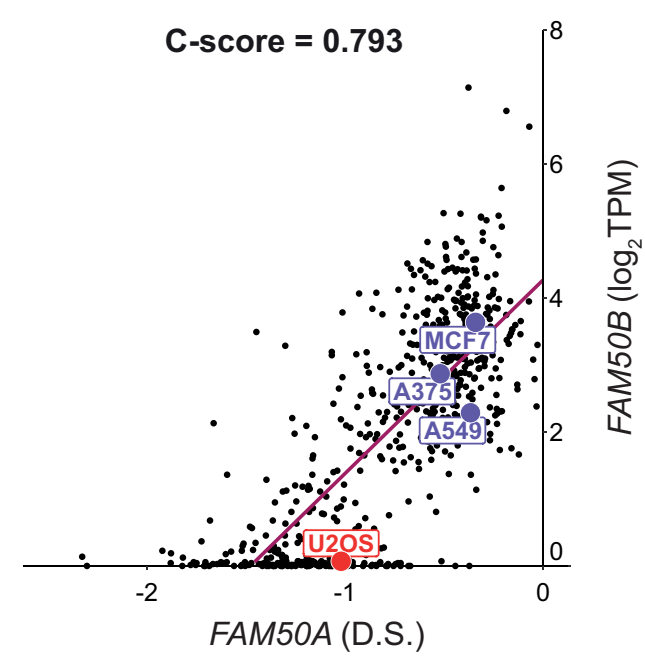

C

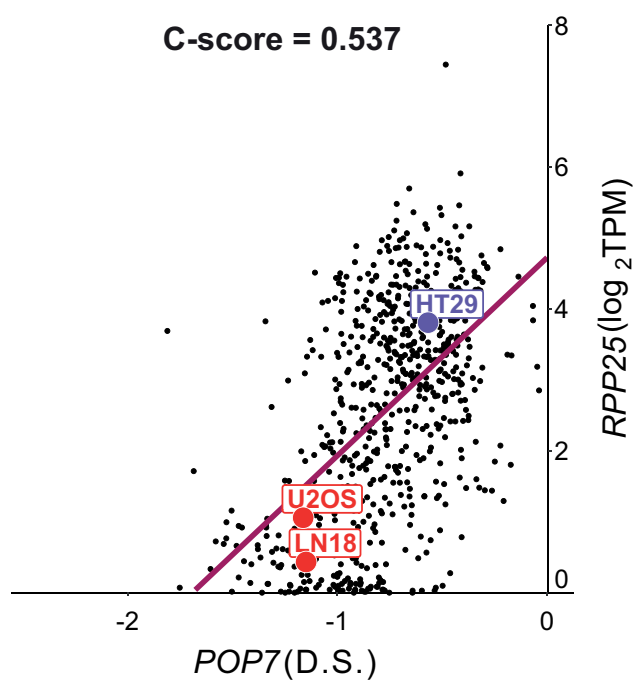

B

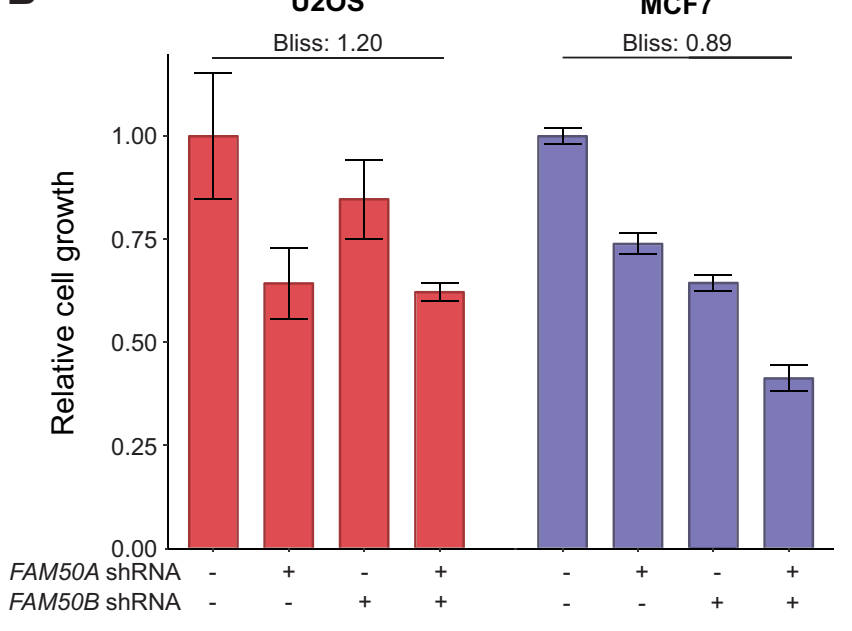

D

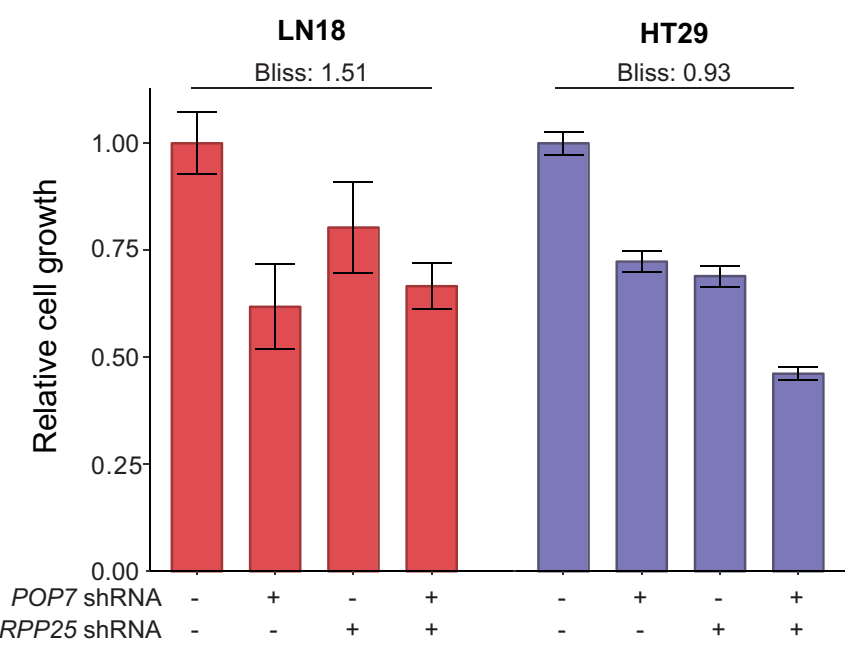

Figure 3. Experimental validation of cell-specific expression buffering between FAM50A and FAM50B, and POP7 and RPP25. (A) C-score plot of the highest C-score gene pair, FAM50A (dependency score-D.S.) and FAM50B (G2) expression, labeled with the U2OS (predicted not synergistic) and MCF7 (predicted synergistic) cell lines. (B) Relative cell growth between 0 and $90 \mathrm{~h}$ based on fold-change in confluency of the U2OS and MCF7 cell lines with or without FAM50A or FAM50B suppression. Bliss scores indicate strength of synergy between double suppression of FAM50A and FAM50B compared to either gene alone. Error bars indicate standard deviation of six technical repeats. (C) C-score plot of the non-duplicated gene pair, i.e., POP7 dependency score and RPP25 gene expression, labeled with the LN18 (predicted not synergistic) and HT29 (predicted synergistic) cell lines. (D) Relative cell growth between 0 and $90 \mathrm{~h}$ based on fold-change in confluency of the HT29, U2OS and LN18 cell lines with or without shRNA-based POP7 or RPP25 suppression. Bliss scores indicate strength of synergy between double suppression of $P O P 7$ and RPP25 compared to either gene alone. Error bars indicate standard deviation of six technical repeats.

the cell lines at the top-right of the plot would be more sensitive to dual suppression of FAM50A and FAM50B. We used gene-specific small hairpin RNAs (shRNAs) to suppress expression of FAM50A and FAM50B, individually and in combination. The suppressive effects of the shRNAs were verified using RT-qPCR (Supplementary Fig. S5C). Consistent with our expectations, we observed stronger growth suppression in the MCF7 cell line relative to the U2OS cell line in two experimental repeats (Fig. 3B, Supplementary Fig. S5A,B). An additional cell line, A549, located at the middle of the C-score plot, however, showed a medium growth suppression relative to MCF7 and U2OS cells (Supplementary Fig. S5A,B). Next, we quantified FAM50A and FAM50B genetic interactions in these different cell lines by Bliss score ${ }^{41}$, where scores lower than one implies stronger synergistic interactions (see "Methods"). Indeed, the FAM50A-FAM50B gene pair in MCF7 cell lines was calculated to have a potential synergistic effect, whereas the calculation showed no synergistic effect in the U2OS cell line (Bliss score: 0.89 in MCF7 and 1.20 in U2OS). Importantly, a recent study focusing on genetic interaction of duplicated 
genes identified the FAM50A and FAM50B gene pair as the most significant interacting duplicated gene pair in the human genome ${ }^{16}$, further supporting the inference power of our C-score index. Moreover, the A375 cell line was used in that recent study, and it is predicted to display strong synergy based on our C-score plot of FAM50A and FAM50B (Fig. 3A).

Although duplicated genes are well recognized for their buffering relationship, there is limited evidence supporting intrinsic buffering among non-duplicated genes. Thus, we sought to experimentally examine a pair of non-duplicated genes with a high C-score, so we targeted the $P O P 7-R P P 25$ pair. These two genes encode protein subunits of the ribonuclease P/MRP complex. In the C-score plot of POP7-RPP25 (Fig. 3C), the HT29 cell line lies in the top-right region and the U2OS and LN18 cell lines are in the bottom-left region, inferring a likelihood for a stronger buffering effect in the HT29 cell line. To validate buffering effects, we suppressed expression of $P O P 7$ and RPP25 using gene-specific shRNAs in these three cell lines (verified using RT-qPCR, Supplementary Fig. S5F). Since expression of $R P P 25$ is too low to be quantified using RT-qPCR, we validated suppression of $R P P 25$ using western blot (Supplementary Fig. S6). Consistent with the C-score plots, we observed that dual suppression of POP7 and RPP25 corresponded with calculations of strong synergistic effects for the HT29 cell line but not for the U2OS or LN18 cell lines (Bliss scores for POP7-RPP25 genetic interactions are 0.931 in HT29 and 1.51 in LN18, shown in Fig. 3D; and Bliss score of 1.03 in U2OS is shown in S5D. Experimental repeat shown in Supplementary Fig. S5E). Collectively, the results suggest that C-score-inferred buffering gene pairs can be non-duplicated functional analogs in the same protein complex or duplicated genes of the same family.

Tissue specificity of CEBU. One key feature of intrinsic buffering is cross-cell variation in the expression of buffering genes (G2s) in conjunction with cell-specific dependency of the buffered genes (G1s) (Fig. 1). We hypothesized that the source of this cross-cell variation in G2 expression is embedded in the distinct transcriptional programs of different tissues. Therefore, we examined if the expression of high C-score G1s and G2s is tissue-specific. We calculated a tissue specificity index, $\tau^{42}$, for each gene to establish if it displays low (low $\tau$, broadly expressed across tissues) or high tissue specificity (high $\tau$, only expressed in one or a few specific tissues). As shown in Fig. 4A, G2s generally presented higher tissue specificity compared to G1s (significant with $t$-test, $p<2.2 \mathrm{e}-16$ ) and compared to the control generated by randomly shuffling G2s across cell lines (Supplementary Fig. S7A). Together, these results indicate that G1s are generally expressed in the majority of cell types, whereas expression of G2s is more tissue-specific.

The pronounced tissue-specificity of G2 expression supports the cell-specific buffering concept proposed by CEBU and may be extended to tissue-specific intrinsic buffering. To further explore tissue distribution of the CEBU concept, we generated normalized C-score plots for all high C-score gene pairs whereby the G1 dependency scores across all cell lines were quantile-normalized to be between - 1 and 0 and the G2 expression values were normalized to be between 0 and 1 (Fig. 4B). We plotted these values against each other and then divided the resulting plot into nine equal regions by radiating lines out from zero (R1 to R9, Fig. 4B). As per the examples suggest in Fig. 3A,C, we hypothesized that there would be specific tissue types enriched in the regions R1-R4, which we speculated to display stronger CEBU-mediated buffering capacity. Similarly, there would be enrichment of specific tissue types in regions R6-R9, which we speculated to infer lower buffering capacity. Accordingly, considering a total of 29 tissue/cell types, we calculated the proportion of each tissue/cell type in each region of the plot in Fig. $4 \mathrm{~B}$, as well as the percentage of CEBU-enriched gene pairs for each tissue/ cell type (see "Methods"). For each plot region, we indeed observed specific enrichment in one to three tissue/ cell types presented a high percentage of CEBU-enriched gene pairs (Fig. 4C and Supplementary Fig. S7B). For example, for region R1, 98.0\% of the CEBU-enriched gene pairs are highly expressed in cells derived from bone tissue (Fig. 4C), whereas region R9 encompasses a high percentage of strongly-expressing CEBU-enriched gene pairs in blood cells (lymphoma: 13.9\%, leukemia: 10.6\%, and multiple myeloma: $9.2 \%$ Fig. 4C). We also noted a few reoccurring tissue/cell types across regions speculated to infer high buffering capacity (central nervous system in R2, R3, and R4) or in low buffering regions (leukemia in R7, R8, and R9; lymphoma in R8 and R9) (Fig. 4C), indicating that particular tissue/cell types are with specific distributions given a CEBU setting. These results strengthen that $\mathrm{CEBU}$ may be capable of reflecting tissue-specific intrinsic buffering, and that whereas buffered G1s are generally expressed across tissue types, the buffering G2s are expressed in specific tissue/cell types, potentially contributing to tissue-specific functions.

Harnessing C-score to calculate the buffering capacity of CEBU. As suggested by our experimental results in Fig. 3, cell lines located in the upper right of a C-score plot are more sensitive to dual gene suppression, indicating a possibility of higher buffering capacity from G2s. Adding to this notion, specific tissues tend to be enriched at the upper right of the C-score plot (Fig. 4), prompting investigation on cell/tissue-specific buffering capacity. To quantify G2 buffering capacities in various cells or tissues, we calculated buffering capacities as the relative G2 expression (compared to that of all other cell lines) of the cell line of interest adjusted by the C-score of the gene pair (Fig. 5A and "Methods"). We hypothesized that the C-score-derived buffering capacities can be an indirect inference of functional buffering and genetic interaction. Hence, we sought to validate these C-scorederived buffering capacities between G1 and G2 using experimental results from four independent studies in human cells (Supplementary Table S3) ${ }^{19,32,43,44}$. The four studies are described as follows: Rosenbluh et al. investigated the genetic interaction map of beta-catenin-active and beta-catenin-inactive cancers with combinatorial CRISPR screening in four cancer cell lines ${ }^{43}$. Shen et al. examined 73 gene pairs in combination $(141,912$ interactions) in three different cell lines ${ }^{32}$. With a more robust CRISPR screening approach, Najm et al. performed pairwise combinations of 158 genes knockout in six cancer cell lines ${ }^{44}$. Focusing on metabolic genes, Zhao et al. did a combinatorial CRISPR screening in two cell lines ${ }^{19}$. Using the receiver operating characteristic (ROC) curve to assess the performance of buffering capacity predictions, we observed that the resulting area under curve 

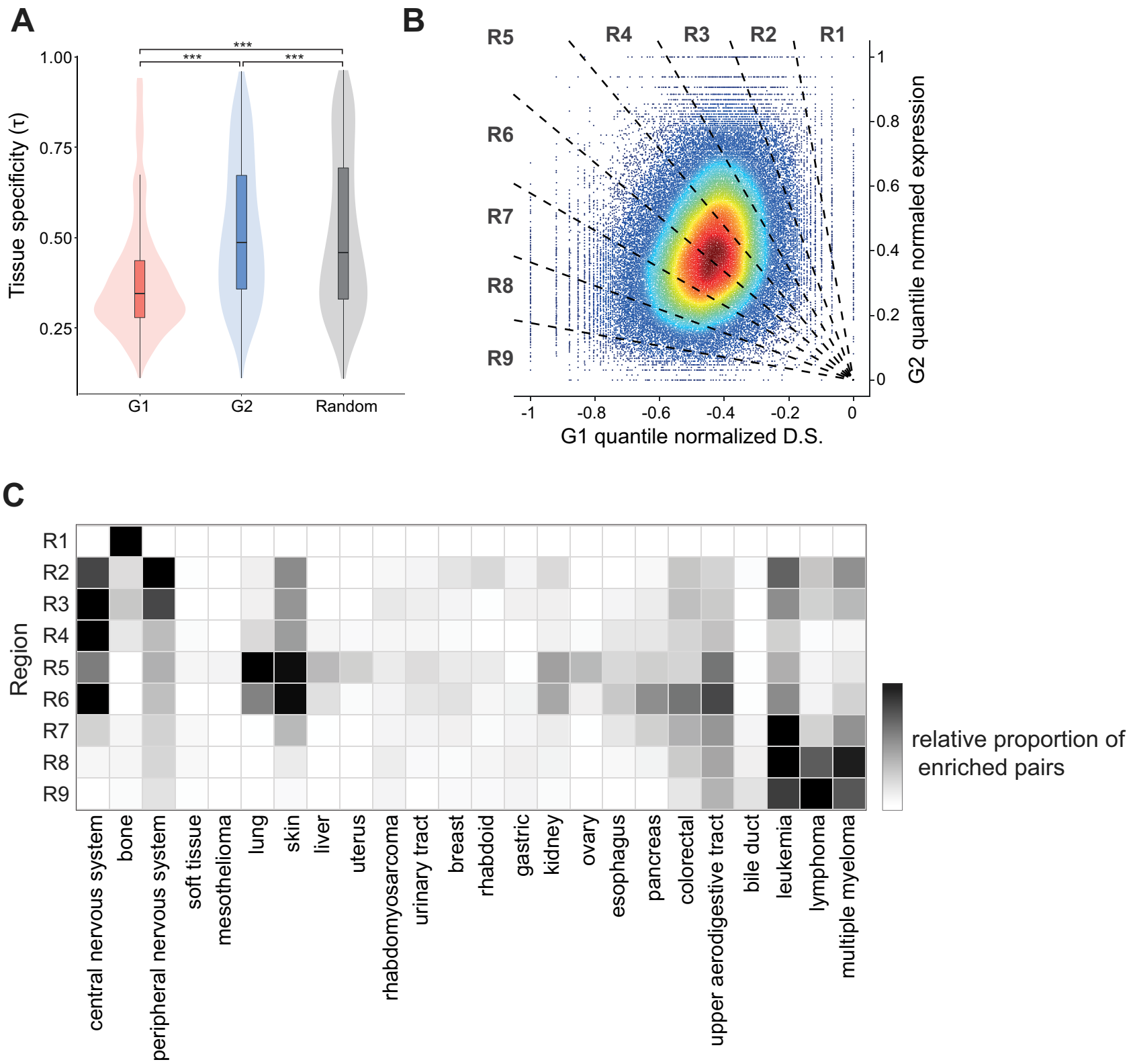

Figure 4. Tissue specificity of CEBU. (A) Tissue specificity $(\tau)$ of G1 and G2 pairs. $\tau$ was calculated for G1 and G2 from high C-score gene pairs. Statistical significance was assessed by paired- $t$ test. (B) Density plot showing overlay of 100,000 randomly selected high C-score gene pairs. D.S. (G1s) and expression (G2s) were normalized to be between -1 and 0 or 0 and 1, respectively. Each dot represents a cell line based on its normalized D.S. for G1 and normalized expression for G2. Color gradient indicates low to high density as blue to dark red, respectively. The normalized C-score plot was divided equally into nine regions (R1-R9) by radiating lines out from zero. (C) Tissue/cell-type specificity of each region (R1-R9) of the normalized C-score plot. The heatmap represent the relative percentage of statistically enriched gene pairs for the corresponding tissue/cell types. The heatmap is created by the Conditional Formatting function of Excel.

(AUC) is significantly larger than random (Mann-Whitney $U$ test with false discovery rate, FDR $<0.1$, Fig. 5B). Furthermore, predictive performance increased for higher C-score cutoffs, as indicated by their increasing AUC (Fig. 5B). Moreover, the buffering capacity is quantitatively correlated with the strength of genetic interaction. We observed a negative correlation between C-score-derived buffering capacities and experimentally validated genetic interactions $(\mathrm{C}$-score cutoff $=0.25$, correlation $=-0.231, p$-value $=0.034, \mathrm{FDR}=0.081$, Supplementary Fig. S8A), and this correlation is stronger for higher C-score cutoffs (Supplementary Fig. S8B). Even though this correlation coefficient of -0.231 is not strong (although it is statistically significant), the intrinsic variability associated with collating experimental results from four independent studies must be considered a contributory factor to weakening that correlation ${ }^{19,32,43,44}$. Moreover, predictions of genetic interactions based on CEBU buffering capacity are robust even when different thresholds for calculating buffering capacity are applied ("Methods" and Supplementary Fig. S8C). Accordingly, CEBU buffering capacity may be used to infer genetic 
A

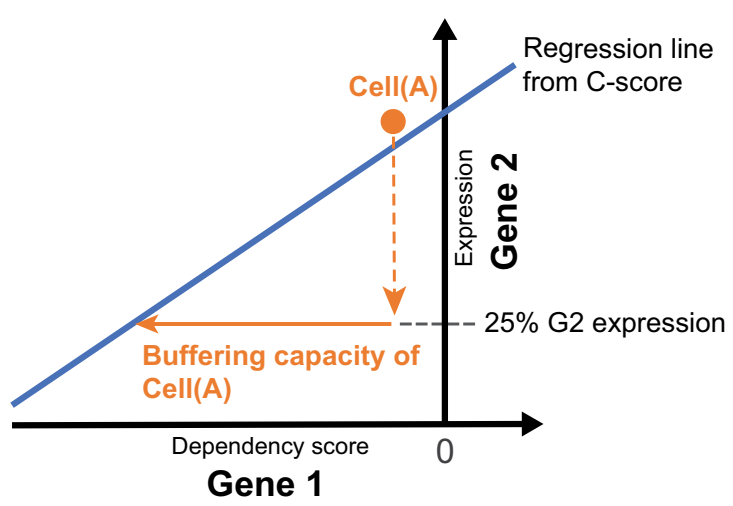

B

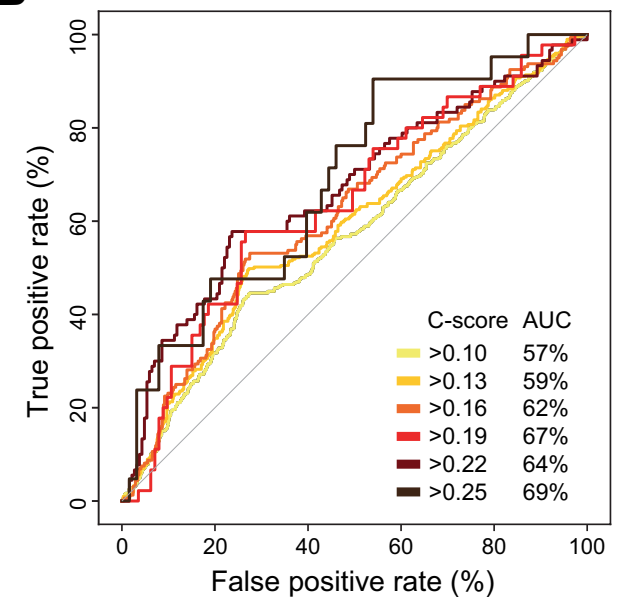

\section{C}

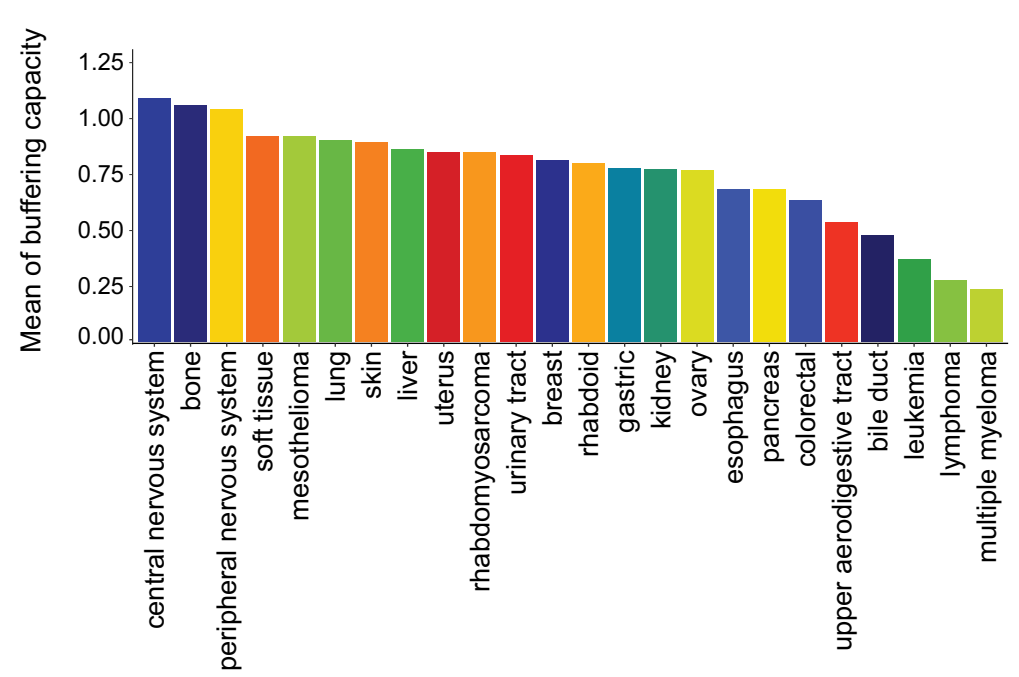

Figure 5. C-score-derived tissue-specific buffering capacity. (A) Illustration showing how cell-specific buffering capacities were derived from C-scores. Buffering capacity was calculated based on: (1) the regression line of the C-score for the gene pair; and (2) relative G2 expression (compared to that of all other cell lines) for the cell line of interest. See "Methods" for the formula for buffering capacity calculation. (B) Predictive performance shown as ROC curves for predicting genetic interactions using cell-specific buffering capacity. Prediction sets consist of 84 data-points ( 37 unique genetically interacting gene pairs) across 8 cell lines with a C-score cut-off of 0.25 . (C) Mean buffering capacity for each tissue type (lower panel) and the corresponding proportion of enriched gene pairs for each region (upper panel).

interactions in human cells. Since CEBU may be reflective of tissue-specific intrinsic buffering (Fig. 4), we also quantified buffering capacity in various tissue/cell types. We calculated the average buffering capacity for each tissue/cell type based on high C-score gene pairs (Fig. 5C). In line with our enrichment analysis presented in Fig. 4, the top three most buffered tissues are the central nervous system, bone and the peripheral nervous system. In contrast, blood cells-including multiple myeloma, lymphoma, and leukemia cell lines-exhibited the lowest buffering capacities.

CEBU-mediated buffering capacity is indicative of cancer aggressiveness. Inspired by the protooncogenes we identified according to C-scores (Fig. 2E), we wondered if cancers in various tissues may take advantage of the buffering capacities endowed by the CEBU mechanism for robust proliferation. In other words, would higher CEBU-mediated buffering capacity render cancers more robust and aggressive, thereby resulting in a poorer prognosis? To test this hypothesis, we established a "ground-truth" of expression-based cancer patient prognosis by analyzing patient gene expression and survival data for all 30 available cancer types from The Cancer Genome Atlas (TCGA) ${ }^{45}$. Here, we assessed differential patient survival against gene expression using Cox regression and controlling for clinical characteristics including age, sex, pathological stage, clinical stage, and tumor grade, followed by multiple testing correction $(\mathrm{FDR}<0.1)$. Then, we examined the performance of CEBU-mediated buffering capacity in terms of predicting the ground-truth dataset. As an example, in Fig. 6A we present potential buffering to $N A M P T$ of the $\mathrm{NAD}^{+}$salvage pathway, where cancers may be addicted to 
A

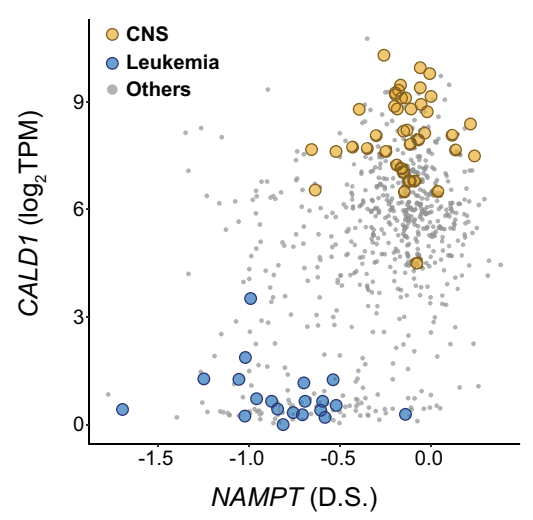

C ${ }_{0.8}$ Patient survival

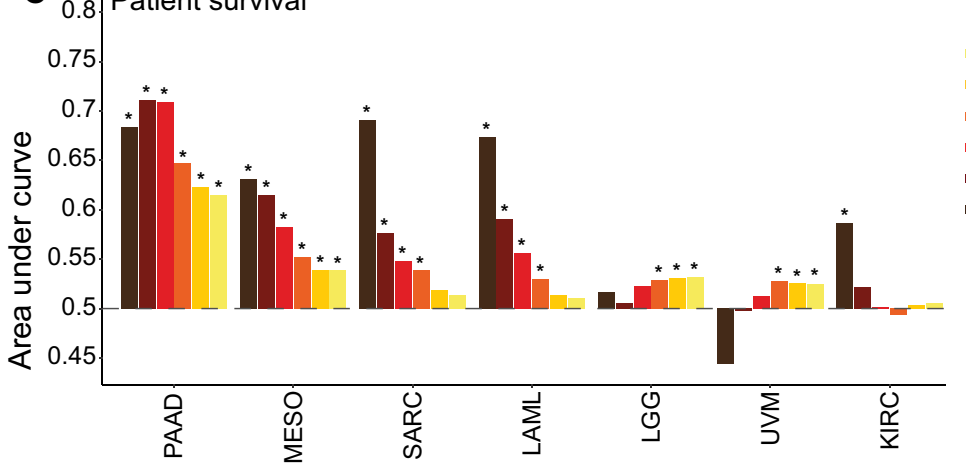

B
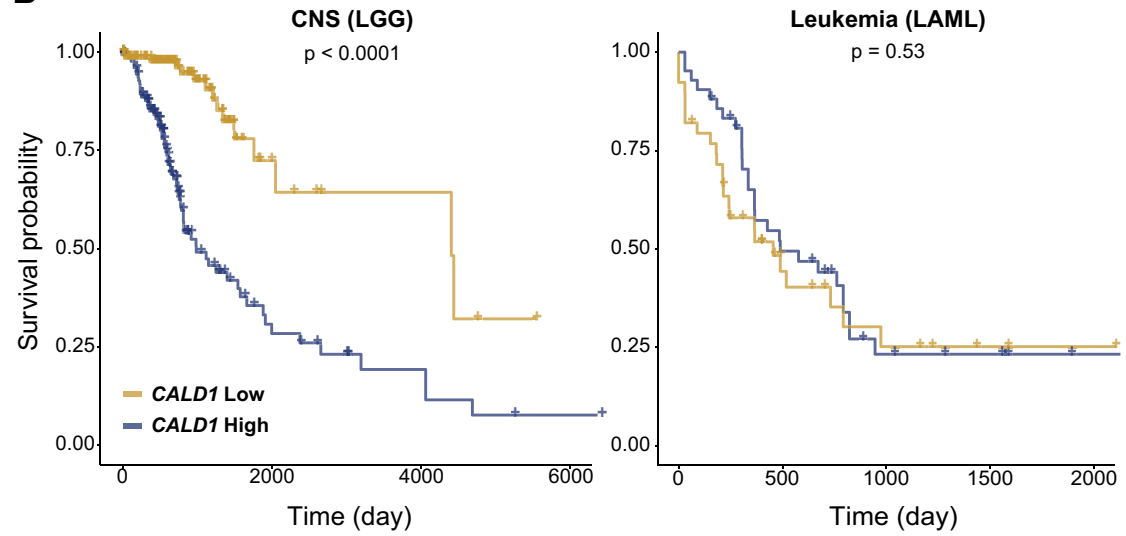

\section{C-score}

$>0.15$

$>0.2$

$>0.25$

$->0.3$

$->0.35$

$>0.4$

Figure 6. Harnessing cell-specific high C-score gene pairs for cancer patient prognosis. (A) C-score plot of NAMPT dependency score and CALD1 gene expression $(\mathrm{C}$-score $=0.447)$. Yellow circles represent central nervous system (CNS) cell lines and blue circles denote leukemia cell lines. (B) Kaplan-Meier overall survival plots for CNS (LGG, lower grade glioma, left panel) and leukemia (LAML, acute myeloid leukemia, right panel) cancer patients. Patients were stratified by high $(>75 \%)$ or low $(<25 \%)$ expression of $C A L D 1$, and $p$-values were calculated using Cox regression controlling for age, sex, pathological staging, clinical staging, and tumor grade, and corrected for multiple testing (FDR $<0.1)$. (C) AUC of ROC curves based on C-score gene pair-based prediction of survival for each cancer type with different C-score cutoffs. Only the cancer types with at least one significantly positive C-score cutoff and those containing more than 50 positive genes predicting patient survival with statistical significance are shown $\left({ }^{*}\right.$ denotes $\left.p<0.05\right)$.

this pathway ${ }^{46}$. We discovered that the NAMPT-CALD1 gene pair, comprising the NAMPT dependency score and CALD1 gene expression, demonstrate a high C-score of 0.446 , and its CEBU-mediated buffering capacity is high in CNS but low in blood cells. When we stratified patients based on CALD1 expression, we observed a considerable difference in survival for patients suffering lower grade glioma (LGG-a cancer of the CNS, see Supplementary Table S4 for cross-referencing between cell lines and TCGA cancers and for the full names of cancer abbreviations), but not for patients with acute myeloid leukemia (LAML—a cancer of the blood, Fig. 6B left panel for LGG and right panel for LAML). Mean CEBU-mediated buffering capacity for the NAMPT:CALD1 gene pair is 1.47 in the CNS (i.e. tissue/cell types displaying strong buffering capacity), but only -0.88 in leukemic blood cells (i.e. exhibiting weak buffering capacity) (Fig. 6A). Thus, based on our ground-truth dataset, the buffering capacity of the NAMPT and CALD1 gene pair in different tissue/cell types can be used to predict patient survival for specific cancer types.

We systematically assessed how buffering capacity from C-score-identified gene pairs could help predict cancer patient survival for all 30 TCGA cancer types. We found that for 15 of those cancers, at least $1 \%$ of genes across the genome can predict patient survival (with statistical significance assessed by Mann-Whitney $\mathrm{U}$ test), and for 7 of these 15 cancer types, the performance of CEBU-mediated buffering capacity for at least one C-score cutoff was significantly better than random (AUC $>0.5, F D R<0.1)$ (Fig. 6C). In general, buffering capacity-based predictions performed better for higher C-score cutoffs. Taken together, our results show that the CEBU-mediated buffering capacity derived from our C-score index can be indicative of cancer aggressiveness, as illustrated by patient survival.

\section{Discussion}

In multicellular organisms, different cells and tissues conduct various functions via specialized cellular structures and/or according to specific states (e.g., signaling and/or metabolic states) by regulating cell- and tissue-specific gene expression. Our study proposes that this cell- and tissue-specific gene expression not only contributes directly to tissue-specific functions, but also allows buffering for functional enhancement. This type of functional 
buffering, which we have termed cell-specific expression buffering (CEBU), suggests essential functions of a gene can be buffered by increased expression of another gene. Accordingly, essentiality of broadly expressed genes, such as housekeeping genes, are coupled with increased expression of another gene, potentially maintaining housekeeping functions in specific tissues. Furthermore, the CEBU relationship appears to be especially prevalent in tissues of low regenerative capacity (e.g., bone and neuronal tissues) and it can stratify cancer patient survival, inferring tumor aggressiveness. Although functional buffering has long been known as critical to biological robustness, the mechanisms underlying functional buffering remain largely unknown ${ }^{3}$. The proposed CEBU concept represents a possible buffering mechanism in multicellular organisms that is critical for tissue homeostasis and cancer robustness.

One key feature of CEBU is the distinct patterns of expression and dependency (essentiality) between the buffered genes (G1s) compared to buffering genes (G2). In general, G1s tend to be broadly expressed with stronger dependency, whereas expression of G2s is more tissue-specific and less essential (Fig. 4A and Supplementary Fig. S2C). Generally, the essentiality of genes is correlated with their expression level and tissue specificity ${ }^{47-49}$. Housekeeping genes that are broadly expressed in most cells exhibit stronger essentiality. In contrast, genes expressed in specific cell types are considered to have weaker essentiality. Here, cooperation of housekeeping genes and tissue-specific genes of similar functions may be associated by the proposed CEBU mechanism, enabling regulation of cellular functions via functional buffering across tissue/cell types. Specifically, house-keeping functions like metabolism, transcription, translation, and cell-cycle-related processes are highly enriched among high C-score gene pairs (Fig. 2E), likely inferring that house-keeping functions can be robustly maintained via CEBU-mediated functional buffering.

As a cell- and tissue-specific buffering mechanism, we postulate that CEBU may endow buffering capacity on specific cells/tissues in order to maintain their functions and survival, such that CEBU may compensate for the lack of regenerative capacity in certain tissues. Our analysis shows neuronal and bone tissues to have the strongest CEBU-mediated buffering capacities (Fig. 4C, 5C), both of which exhibit relatively low regenerative capacities $^{50-52}$. In contrast, human blood cells, which are fully regenerated in 4 to 8 weeks ${ }^{53}$, are predicted to have the weakest buffering capacities (Fig. 5C). Therefore, it is tempting to speculate that cell types of weaker regenerative capacities, such as neurons, need to sustain robust cellular functions through buffering. In contrast, highly regenerative tissues are frequently replaced, so they have less need for functional buffering.

Unlike the needs-based buffering mechanism, whereby the buffering gene is only activated when its buffered function is compromised, the CEBU-mediated intrinsic buffering proposed herein maintains a constitutively active state with cell- and tissue-specificity. Since the buffering gene (G2) is continuously expressed, there is no need for a control system to monitor if a function has been compromised and to activate the expression of the buffering genes. As a result, no response time is needed for intrinsic buffering, unlike for needs-based buffering. Intrinsic buffering thus poses an advantage in its capability to buffer housekeeping genes, which is performed constitutively, differing from the needs-based buffering that is mostly characterized as stress-responsive ${ }^{54}$. Accordingly, the proposed intrinsic CEBU mechanism may enable or adjust buffering capacity by regulating the expression of buffering genes via cell- or tissue-specific epigenetic regulators. Overall then, CEBU describes a simple, efficient and potentially versatile mechanism for functional buffering in humans and potentially other multicellular organisms.

We observed an enrichment of duplicated genes among high C-score gene pairs, supporting the notion that duplicated genes contribute to the context-dependent essentiality of their paralogous genes ${ }^{10-12}$. In addition to duplicated genes, our C-score index identified a high percentage of non-duplicated gene pairs with high buffering capacities (Fig. 2B), and these non-duplicated gene pairs tend to belong to the same pathways and/or protein complexes (Fig. 2C,D). Therefore, it is possible that many of these G1s and G2s represent non-orthologous functional analogs. As support, we identified the POP7 and RPP25 gene pair, both are subunits of the ribonuclease P/MRP complex, as potential buffering pairs (Fig. 3C,D, Supplementary Figs. S5D-F, S6). One simple scenario could be that G1 and G2 physically interact with each other to form a protein complex, wherein G1's function can be structurally substituted by G2. More sophisticated and indirect functional buffering can also occur between G1s and G2s given the complex interactions among biological functions ${ }^{55}$. We expect that CEBU exerts buffering effects through additional types of molecular interactions, which remain to be tested experimentally.

Experimental validations of functional buffering remain to be a challenging task. Buffering of one gene may be achieved through multiple mechanisms from multiple genes, leading to difficulties in selecting test targets and types of experiments. Moreover, functional buffering may be different across cell lines, which is in line with the proposed CEBU mechanism. Using CEBU as a base provides an approach to select for target gene pairs and cell lines and can test for buffering via expression. We have demonstrated the validity of using the C-score plot to identify functional buffering in two pairs of genes, FAM50A-FAM50B and POP7-RPP25 through shRNAsilencing (Fig. 3, Supplementary Figs. S5, S6). However, the type of experiment remains to be a technical challenge. shRNA-silencing may not fully recapitulate the screening results from CRISPR. We noticed a discrepancy between DepMap screening data (average D.S. of FAM50B in A549 is 0.256) and our own validation results regarding the essentiality of FAM50B in A549 (Supplementary Fig. S5C). This discrepancy could be due to the incompleteness of shRNA-mediated gene silencing, leaving a low expression level of targeted endogenous genes. This low level of expression may trigger secondary responses that leads to different phenotypic outcomes. Nevertheless, our results showed potential synergistic effects upon dual suppression in predicted sensitive cells lines as oppose to single knockdowns (Fig. 3, Supplementary Figs. S5, S6), though other experimental methods, such as CRISPR, may provide additional validation.

C-score-derived cell-specific buffering capacities comply well with experimentally validated genetic interactions in human cells (Fig. 5B and Supplementary Fig. S8), indicating that CEBU may potentially represent a critical mechanism for synthetic lethality in human cells. However, accurate inference of synthetic lethality is difficult as all computational predictions inevitably generate false positives. Despite a stringent selection with 
low $p$-values and controls for multiple testing (Fig. 1C), it remains possible that some high C-score gene pairs are resultant of random chance. Validation of synthetic lethality interaction remains a daunting challenge in practice. To systematically characterize genetic interactions in organisms with complex genomes due to large numbers of possible gene pairs, i.e. 200 million gene pairs in humans. Another method to infer authenticity of synthetic lethality is of recurring computational predictions across studies. However, predictions emanating from different studies exhibit little overlap ${ }^{30}$, evidencing the marked complexity of synthetic lethality in humans. It is possible that each of the current predictions focuses on one or a few characteristics of synthetic lethality such that assembling them does not reveal the same predictions. In this sense, the CEBU mechanism proposed here can contribute both experimentally and computationally to a better characterization of human genetic interactions.

Using G2 expression of a high C-score gene pair to stratify cancer patients, we observed a significant difference in cancer patient survival, indicating that stronger CEBU-mediated buffering capacity could be indicative of cancer aggressiveness in patients (see Fig. 6A,B for an example). Indeed, buffering capacity helped predict cancer patient survival in 7 of 15 cancer types and, generally, the performance was better for higher $\mathrm{C}$-score cutoffs (Fig. 6C). This result supports our hypothesis that stronger buffering capacity via higher G2 expression contributes to cancer robustness in terms of proliferation and drug resistance. Prognosis in cancer patients can be affected by multiple factors, and some of them are unmeasurable and not accounted for in current study. However, given the complexity of cancers, it is surprising to see the results possibly suggests a general predictivity of cancer prognosis by individual high C-score gene pairs. We suspect that some cancer cells may adopt this cell- and tissue-specific buffering mechanism to enhance their robustness in proliferation and stress responses by targeting the expression of buffering genes. Clinically, the expression of such buffering genes could represent a unique feature for evaluating cancer progression when applied alongside other currently used clinical characteristics. Finally, experimental validation of C-score-predicted genetic interactions will help identify potential drug targets for tailored combination therapy against specific cancers.

\section{Methods}

Retrieval and processing of dependency score and gene expression data. Data on dependency scores and CCLE (Cancer Cell Line Encyclopedia) gene expression were downloaded from the DepMap database (DepMap Public 19Q4) ${ }^{14,15}$. Dependency scores modeled from the CERES computational pipeline based on a genome-wide CRISPR loss-of-function screening were selected. CCLE expression data was quantified as $\log _{2}$ TPM (Transcripts Per Million) using RSEM (RNA-seq by Expectation Maximization) with a pseudo-count of 1 in the GTEx pipeline (https://gtexportal.org/home/documentationPage). Only uniquely mapped reads in the RNA-seq data were used in the GTEx pipeline. Integrating and cross-referencing of the dependency score and gene expression datasets yielded 18,239 genes and 684 cell lines. Genes lacking dependency scores for any one of the 684 cell lines were discarded from our analyses.

C-score calculation. Our C-score index integrates the dependency scores of buffered genes (G1) and the gene expression of buffering genes (G2) to determine the buffering relationship between gene pairs. Genes with mean dependency scores $>0$ or mean gene expression $<0.5 \log _{2}$ TPM were discarded, yielding 9196 G1s and 13,577 G2s. The C-score integrates the correlation $(\rho)$ and slope between the dependency score of gene $G 1$ and the gene expression of gene $G 2$, defined as:

$$
\text { C-score }=\rho_{G 1, G 2}\left(1+b \frac{\text { slope }_{\min }}{\text { slope }_{G 1, G 2}}\right),
$$

where $\rho$ denotes the Pearson correlation coefficient and slope $_{\min }$ denotes the minimum slope of all considered gene pairs that present a statistically significant positive correlation, which is 0.00748 in this analysis. The normalized slope can be weighted by cell- and tissue-type specific $b$. In this analysis, $b$ is set as 1 for a pan-cell or pan-cancer analysis.

Construction of C-score null distribution. As a control, expression of each gene was randomly shuffled amongst the 684 cell lines. The shuffled expression values were used in place of true expression in the C-score calculation to create a randomly shuffled null distribution. Five shuffled expression datasets were generated to calculate five null distributions, one of which is shown.

Duplicated gene assignment. Information on gene identity was obtained from ENSEMBL (release 98, reference genome GRCh38.p13) ${ }^{56}$. Two genes are considered duplicated genes if they have diverged from the same duplication event.

Enrichment analysis for buffering gene pairs. For enrichment analysis of duplicated gene pairs, we conducted hypergeometric test. Given a specified C-score cutoff, the test assesses statistical significance by calculating the proportion of duplicated gene pairs higher than cutoff among all duplicated gene pairs, and then compare to the proportions of all high C-score pairs higher than cutoff among all high C-score pairs.

For enrichment analysis of same function or signaling pathways, we adopted a previously described methodology $y^{57}$. Briefly, GO and KEGG gene sets were downloaded from the Molecular Signatures Database (https://www.gsea-msigdb.org/gsea/msigdb/). The number of total possible gene pairs is 9196 (G1) $\times 13,577$ (G2). The condition of G1 and G2 being the same gene was excluded as a potential buffering gene pair under all C-score cutoffs. Enrichment was calculated as: 


$$
\log \frac{\frac{e_{a c}}{e_{a}}}{\frac{e_{c}}{e_{t}}},
$$

where $e_{a c}$ represents the number of gene pairs that are both annotated and with buffering capability, $e_{a}$ is the number of annotated gene pairs, $e_{c}$ is the number of buffering gene pairs, and $e_{t}$ is the total number of gene pairs.

Protein-protein interaction (PPI) data was downloaded from the STRING database (version 11) ${ }^{39}$. Only high-confidence interactions (confidence $>0.7$ ) in human were considered. The STRING database determines confidence by approximating the probability that a link exists between two enzymes in the KEGG database. Data on protein core complexes were downloaded from CORUM (http://mips.helmholtz-muenchen.de/corum). The enrichment calculation is the same as for GO and KEGG, except that $e_{a c}$ represents the number of gene pairs that have PPI or are in the same complex and have buffering capability, and $e_{a}$ is the number of gene pairs that have PPI or are in the same complex.

Construction of our human buffering gene network. The directional human buffering gene network was constructed from gene pairs exhibiting high C-scores $(>0.25)$. For illustration, isolated subnetworks are not shown. We visualized the network using Cytoscape (https://cytoscape.org/) and MATLAB. GO enrichment was conducted on each cluster using g:Profiler ${ }^{58}$. To identify functionally-related gene clusters in the human buffering gene network, the genes with enriched functions were inputted into the SAFE algorithm ${ }^{59}$. The neighbor radius was determined by regional enrichment of sub-networks for each GO-enriched function.

Experimental validation. A549, HT29, LN18, MCF7, and U2OS cell lines were selected based on their distribution across the C-score plots (Fig. 3A,C), indicating different buffering capacities. All cell lines were purchased from ATCC and they were cultured in Dulbecco's Modified Eagle Media (LN18), Ham's F-12K Medium (A549), or RPMI 1640 media (HT29, MCF7, and U2OS) supplemented with 5\% fetal bovine, serum, 100 U/ $\mathrm{mL}$ penicillin, $100 \mu \mathrm{g} / \mathrm{mL}$ streptomycin, and $250 \mathrm{ng} / \mathrm{mL}$ fungizone (Gemini Bio-Products). Cell growth was monitored by time-lapse imaging using Incucyte Zoom, taking images every $2 \mathrm{~h}$ for $2-4$ days. To suppress FAM50A, FAM50B, POP7 and RPP25 expression, lentivirus-based shRNAs were delivered individually or in combination. The gene-specific shRNA sequences are: FAM50A-CCAACATTGACAAGAAGTTCT and GAG CTGGTACGAGAAGAACAA; FAM50B-CACCTTCTACGACTTCATCAT; POP7-CTTCAGGGTCACACC CAAGTA and CGGAGACCCAATGACATTTAT; and RPP25-CCAGCGTCCAAGAGGAGCCTA. To ensure better knockdown of gene expression, shRNAs were delivered twice ( 7 days and 4 days before seeding). Equal numbers of cells were seeded for cell growth measurements by time-lapse imaging using Incucyte Zoom. The lentivirus-based shRNAs were purchased from the RNAi core of Academia Sinica. The growth rate under each condition was measured by fitting cell confluence to an exponential growth curve using the Curve Fitting Toolbox in MATLAB.

Bliss independence model. Cytotoxic synergy was measured using the Bliss independent model ${ }^{41}$. The Bliss model is presented as a ratio of the expected additive effect to the observed combinatorial effect:

$$
E_{\text {bliss }}=\frac{E_{A}+E_{B}-E_{A} \times E_{B}}{E_{A B}},
$$

where $E$ is the effect of $\operatorname{drug} A, B$, or a combination of $A$ and $B$. A ratio of lower than one indicates potential synergistic effect, while larger than one indicates no synergistic effect. Effect was measured by the relative cell growth, based on the fold-change of confluency between 0 and final hours upon suppression of FAM50A and FAM $50 B$ or suppression of POP7 and RPP25 in all cell lines.

Cell-specific buffering capacity and comparison to experimental genetic interactions. Cellspecific buffering capacity was derived from the $\mathrm{C}$-score of a given gene pair and gene expression of the buffering gene (G2) in the cell line of interest following the equation:

$$
\begin{gathered}
\text { buffering capacity }=\frac{\text { cell line expression }-25 \text { th percentile of all expression }(\mathrm{G} 2)}{s l o p e_{\text {mod }}}, \\
\text { where } \text { slope }_{\text {mod }}=\mathrm{C} \text {-score } \times \frac{s d(G 2 \text { expression })}{s d(G 1 \text { dependency })}
\end{gathered}
$$

where $s d=$ standard deviation. The 25th percentile cutoff for expression is determined empirically, although different percentile cutoffs do not qualitatively affect the measurements of buffering capacities (Fig. 5A).

Combinatorial CRISPR screen-derived genetic interaction scores were pooled from four literature sources $^{19,32,43,44}$ (Supplementary Table S3). We only considered cell lines that appear in DepMap CERES 19Q4. There were two C-scores for each gene-pair of the experimental dataset (either gene could be a G1), and we assigned the higher $\mathrm{C}$-score for that gene-pair. Overall, we curated 10,222 genetic interaction scores in various cell lines from the literature, and 1986 out of 10,222 genetic interaction scores had a C-score $>0.1$. To evaluate the validity of buffering capacity, we generated a ground-truth dataset by assigning gene-pairs with a positive genetic interaction as false for buffering and a negative genetic interaction as true for buffering. The qualitative performance of buffering capacity against this ground-truth dataset was assessed by ROC curve. Additionally, we 
correlated the buffering capacity directly via a ground-truth genetic interaction score for quantitative evaluation. We calculated the false discovery rate (FDR) using the Benjamini-Hochberg procedure with a threshold $<0.1$.

Tissue specificity. To calculate tissue-specificity, cell lines were grouped by their respective tissues, and expression of genes in cell lines of the same tissue were averaged. Tissue specificity was calculated as tau $(\tau)^{42}$, where $\tau$ is defined as:

$$
\tau=\frac{\sum_{i-1}^{N}\left(1-\frac{x_{i}}{x_{\max }}\right)}{N-1},
$$

with $N$ denoting the number of tissues, $x_{i}$ denoting the expression of a gene, and $x_{\max }$ denoting the highest gene expression across all tissues. Note, expression values were $\log$-transformed, so $\log _{2} \mathrm{TPM}<1$ was considered as 0 in tissue specificity calculations ${ }^{60}$.

Cancer-specific survival prediction according to C-score gene pairs. Gene expression and survival data from The Cancer Genome Atlas (TCGA) ${ }^{45}$ was retrieved from Xena ${ }^{61}$. The DepMap cancer cell lines were mapped to TCGA cancers based on the annotation in Supplementary Table S4 (cancers that do not have a matched cancer type in CERES 19Q4 were not analyzed). To systematically analyze cancer prognosis, we first performed a multiple test correction on the $p$-values from Cox regression controlling for age, sex, pathological stage, clinical stage and tumor grade. We calculated the FDR using the Benjamini-Hochberg procedure with a threshold $<0.1$. The ground-truth table for each cancer was constructed using the adjusted $p$-value. AUC of ROC curves were used to assess the performance of survival based on buffering capacity. AUCs and ROCs were generated using python and R. The statistical significance of AUC was assessed by Mann-Whitney $U$ test ${ }^{62}$ to evaluate if gene expression with a positive Cox coefficient (poorer prognosis) reflected significantly higher buffering capacities in each cancer with different C-score cut-offs. We excluded the results where there are fewer than 50 positive genes when calculating ROCs. The $p$-values of the Mann-Whitney U test were adjusted using the Benjamini-Hochberg procedure with a threshold $<0.1$.

\section{Data availability}

All C-score gene pairs (https://figshare.com/s/6f8929c6543687a6062f) and programming code (https://figshare. $\mathrm{com} / \mathrm{s} / \mathrm{b} 778489 \mathrm{bb} 2 \mathrm{f} 6 \mathrm{fc} 3 \mathrm{~b} 0069)$ are available in the FigShare repository.

Received: 25 August 2021; Accepted: 3 February 2022

Published online: 22 February 2022

\section{References}

1. Kitano, H. Biological robustness. Nat. Rev. Genet. 5, 826-837. https://doi.org/10.1038/nrg1471 (2004).

2. Masel, J. \& Siegal, M. L. Robustness: Mechanisms and consequences. Trends Genet. 25, 395-403. https://doi.org/10.1016/j.tig.2009. 07.005 (2009).

3. El-Brolosy, M. A. \& Stainier, D. Y. R. Genetic compensation: A phenomenon in search of mechanisms. PLoS Genet. 13, e1006780. https://doi.org/10.1371/journal.pgen.1006780 (2017).

4. Diss, G., Ascencio, D., DeLuna, A. \& Landry, C. R. Molecular mechanisms of paralogous compensation and the robustness of cellular networks. J. Exp. Zool. Part B 322, 488-499. https://doi.org/10.1002/jez.b.22555 (2014).

5. El-Brolosy, M. A. et al. Genetic compensation triggered by mutant mRNA degradation. Nature 568, 193. https://doi.org/10.1038/ s41586-019-1064-z (2019).

6. Kafri, R., Bar-Even, A. \& Pilpel, Y. Transcription control reprogramming in genetic backup circuits. Nat. Genet. 37, $295-299$. https://doi.org/10.1038/ng1523 (2005).

7. Kafri, R., Levy, M. \& Pilpel, Y. The regulatory utilization of genetic redundancy through responsive backup circuits. Proc. Natl. Acad. Sci. USA 103, 11653-11658. https://doi.org/10.1073/pnas.0604883103 (2006).

8. Kafri, R., Springer, M. \& Pilpel, Y. Genetic redundancy: New tricks for old genes. Cell 136, 389-392. https://doi.org/10.1016/j.cell. 2009.01.027 (2009).

9. Koonin, E. V., Mushegian, A. R. \& Bork, P. Non-orthologous gene displacement. Trends Genet. 12, $334-336$ (1996).

10. Wang, T. et al. Identification and characterization of essential genes in the human genome. Science 350, 1096-1101. https://doi. org/10.1126/science.aac7041 (2015).

11. Dandage, R. \& Landry, C. R. Paralog dependency indirectly affects the robustness of human cells. Mol. Syst. Biol. 15, e8871. https:// doi.org/10.15252/msb.20198871 (2019).

12. De Kegel, B. \& Ryan, C. J. Paralog buffering contributes to the variable essentiality of genes in cancer cell lines. PLoS Genet. 15, e1008466. https://doi.org/10.1371/journal.pgen.1008466 (2019).

13. Vu, V. et al. Natural variation in gene expression modulates the severity of mutant phenotypes. Cell 162, 391-402. https://doi.org/ 10.1016/j.cell.2015.06.037 (2015).

14. Ghandi, M. et al. Next-generation characterization of the Cancer Cell Line Encyclopedia. Nature 569, 503. https://doi.org/10.1038/ s41586-019-1186-3 (2019).

15. Meyers, R. M. et al. Computational correction of copy number effect improves specificity of CRISPR-Cas9 essentiality screens in cancer cells. Nat. Genet. 49, 1779. https://doi.org/10.1038/ng.3984 (2017).

16. Thompson, N. A. et al. Combinatorial CRISPR screen identifies fitness effects of gene paralogues. Nat. Commun. 12, 1302. https:// doi.org/10.1038/s41467-021-21478-9 (2021).

17. O'Leary, M. N. et al. The ribosomal protein Rpl22 controls ribosome composition by directly repressing expression of its own paralog, Rpl2211. PLoS Genet. 9, e1003708. https://doi.org/10.1371/journal.pgen.1003708 (2013).

18. Hoffman, G. R. et al. Functional epigenetics approach identifies BRM/SMARCA2 as a critical synthetic lethal target in BRG1deficient cancers. Proc. Natl. Acad. Sci. U. S. A. 111, 3128-3133. https://doi.org/10.1073/pnas.1316793111 (2014).

19. Zhao, D. et al. Combinatorial CRISPR-Cas9 metabolic screens reveal critical redox control points dependent on the KEAP1-NRF2 regulatory axis. Mol. Cell 69, 699-708 e697. https://doi.org/10.1016/j.molcel.2018.01.017 (2018).

20. Gonatopoulos-Pournatzis, T. et al. Genetic interaction mapping and exon-resolution functional genomics with a hybrid Cas9Cas12a platform. Nat. Biotechnol. 38, 638-648. https://doi.org/10.1038/s41587-020-0437-z (2020). 
21. Oike, T. et al. A synthetic lethality-based strategy to treat cancers harboring a genetic deficiency in the chromatin remodeling factor BRG1. Cancer Res. 73, 5508-5518. https://doi.org/10.1158/0008-5472.CAN-12-4593 (2013).

22. Muller, F. L. et al. Passenger deletions generate therapeutic vulnerabilities in cancer. Nature 488, 337-342. https://doi.org/10.1038/ nature11331 (2012).

23. Kranthi, T., Rao, S. B. \& Manimaran, P. Identification of synthetic lethal pairs in biological systems through network information centrality. Mol. Biosyst. 9, 2163-2167. https://doi.org/10.1039/c3mb25589a (2013).

24. Szczurek, E., Misra, N. \& Vingron, M. Synthetic sickness or lethality points at candidate combination therapy targets in glioblastoma. Int. J. Cancer 133, 2123-2132. https://doi.org/10.1002/ijc.28235 (2013).

25. Wang, X. \& Simon, R. Identification of potential synthetic lethal genes to p53 using a computational biology approach. BMC Med. Genom. 6, 30. https://doi.org/10.1186/1755-8794-6-30 (2013).

26. Jerby-Arnon, L. et al. Predicting cancer-specific vulnerability via data-driven detection of synthetic lethality. Cell 158, 1199-1209. https://doi.org/10.1016/j.cell.2014.07.027 (2014).

27. Srihari, S., Singla, J., Wong, L. \& Ragan, M. A. Inferring synthetic lethal interactions from mutual exclusivity of genetic events in cancer. Biol. Direct. 10, 57. https://doi.org/10.1186/s13062-015-0086-1 (2015).

28. Srivas, R. et al. A network of conserved synthetic lethal interactions for exploration of precision cancer therapy. Mol. Cell 63, 514-525. https://doi.org/10.1016/j.molcel.2016.06.022 (2016).

29. Ye, H., Zhang, X., Chen, Y., Liu, Q. \& Wei, J. Ranking novel cancer driving synthetic lethal gene pairs using TCGA data. Oncotarget 7, 55352-55367. https://doi.org/10.18632/oncotarget.10536 (2016).

30. Liu, L. et al. Synthetic lethality-based identification of targets for anticancer drugs in the human signaling network. Sci. Rep. 8, 8440. https://doi.org/10.1038/s41598-018-26783-w (2018).

31. De Kegel, B., Quinn, N., Thompson, N. A., Adams, D. J. \& Ryan, C. J. Comprehensive prediction of robust synthetic lethality between paralog pairs in cancer cell lines. Cell Syst. https://doi.org/10.1016/j.cels.2021.08.006 (2021).

32. Shen, J. P. et al. Combinatorial CRISPR-Cas9 screens for de novo mapping of genetic interactions. Nat. Methods 14, 573. https:// doi.org/10.1038/nmeth.4225 (2017).

33. Bickmore, W. A. The spatial organization of the human genome. Annu. Rev. Genom. Hum. Genet. 14, 67-84. https://doi.org/10. 1146/annurev-genom-091212-153515 (2013).

34. Akdemir, K. C. et al. Disruption of chromatin folding domains by somatic genomic rearrangements in human cancer. Nat. Genet. 52, 294-305. https://doi.org/10.1038/s41588-019-0564-y (2020).

35. Gong, Y. et al. Stratification of TAD boundaries reveals preferential insulation of super-enhancers by strong boundaries. Nat. Commun. 9, 542. https://doi.org/10.1038/s41467-018-03017-1 (2018).

36. Ashburner, M. et al. Gene ontology: Tool for the unification of biology. The Gene Ontology Consortium. Nat. Genet. 25, 25-29. https://doi.org/10.1038/75556 (2000).

37. Kanehisa, M. \& Goto, S. KEGG: Kyoto encyclopedia of genes and genomes. Nucleic Acids Res. 28, 27-30. https://doi.org/10.1093/ nar/28.1.27 (2000)

38. Subramanian, A. et al. Gene set enrichment analysis: A knowledge-based approach for interpreting genome-wide expression profiles. Proc. Natl. Acad. Sci. U. S. A. 102, 15545-15550. https://doi.org/10.1073/pnas.0506580102 (2005).

39. von Mering, C. et al. STRING: Known and predicted protein-protein associations, integrated and transferred across organisms. Nucleic Acids Res. 33, D433-437. https://doi.org/10.1093/nar/gki005 (2005).

40. Ruepp, A. et al. CORUM: The comprehensive resource of mammalian protein complexes-2009. Nucleic Acids Res. 38, D497-501. https://doi.org/10.1093/nar/gkp914 (2010).

41. Bliss, C. I. The toxicity of poisons applied jointly. Ann. Appl. Biol. 26, 585-615 (1939).

42. Yanai, I. et al. Genome-wide midrange transcription profiles reveal expression level relationships in human tissue specification. Bioinformatics 21, 650-659. https://doi.org/10.1093/bioinformatics/bti042 (2005).

43. Rosenbluh, J. et al. Genetic and proteomic interrogation of lower confidence candidate genes reveals signaling networks in betacatenin-active cancers. Cell Syst. 3, 302. https://doi.org/10.1016/j.cels.2016.09.001 (2016).

44. Najm, F. J. et al. Orthologous CRISPR-Cas9 enzymes for combinatorial genetic screens. Nat. Biotechnol. 36, 179-189. https://doi. org/10.1038/nbt.4048 (2018).

45. Hoadley, K. A. et al. Cell-of-origin patterns dominate the molecular classification of 10,000 tumors from 33 types of cancer. Cell 173, 291-304 e296. https://doi.org/10.1016/j.cell.2018.03.022 (2018).

46. Heske, C. M. Beyond energy metabolism: Exploiting the additional roles of NAMPT for cancer therapy. Front. Oncol. 9, 1514. https://doi.org/10.3389/fonc.2019.01514 (2019).

47. Hastings, K. E. M. Strong evolutionary conservation of broadly expressed protein isoforms in the troponin I gene family and other vertebrate gene families. J. Mol. Evol. 42, 631-640. https://doi.org/10.1007/Bf02338796 (1996).

48. Subramanian, S. \& Kumar, S. Gene expression intensity shapes evolutionary rates of the proteins encoded by the vertebrate genome. Genetics 168, 373-381. https://doi.org/10.1534/genetics.104.028944 (2004).

49. Zhang, L. Q. \& Li, W. H. Mammalian housekeeping genes evolve more slowly than tissue-specific genes. Mol. Biol. Evol. 21, 236-239. https://doi.org/10.1093/molbev/msh010 (2004).

50. Iismaa, S. E. et al. Comparative regenerative mechanisms across different mammalian tissues. NPJ Regen. Med. https://doi.org/10. 1038/s41536-018-0044-5 (2018).

51. Fawcett, J. W. The struggle to make CNS axons regenerate: Why has it been so difficult?. Neurochem. Res. 45, 144-158. https://doi. org/10.1007/s11064-019-02844-y (2020).

52. Schmidt-Bleek, K., Petersen, A., Dienelt, A., Schwarz, C. \& Duda, G. N. Initiation and early control of tissue regeneration-Bone healing as a model system for tissue regeneration. Expert Opin. Biol. Ther. 14, 247-259. https://doi.org/10.1517/14712598.2014. 857653 (2014).

53. Biechonski, S., Yassin, M. \& Milyavsky, M. DNA-damage response in hematopoietic stem cells: An evolutionary trade-off between blood regeneration and leukemia suppression. Carcinogenesis 38, 367-377. https://doi.org/10.1093/carcin/bgx002 (2017).

54. DeLuna, A., Springer, M., Kirschner, M. W. \& Kishony, R. Need-based up-regulation of protein levels in response to deletion of their duplicate genes. PLoS Biol. 8, e1000347. https://doi.org/10.1371/journal.pbio.1000347 (2010).

55. Rancati, G., Moffat, J., Typas, A. \& Pavelka, N. Emerging and evolving concepts in gene essentiality. Nat. Rev. Genet. 19, 34-49. https://doi.org/10.1038/nrg.2017.74 (2018).

56. Cunningham, F. et al. Ensembl 2019. Nucleic Acids Res. 47, D745-D751. https://doi.org/10.1093/nar/gky1113 (2019).

57. Boyle, E. A., Pritchard, J. K. \& Greenleaf, W. J. High-resolution mapping of cancer cell networks using co-functional interactions. Mol. Syst. Biol. 14, e8594. https://doi.org/10.15252/msb.20188594 (2018).

58. Raudvere, U. et al. g:Profiler: A web server for functional enrichment analysis and conversions of gene lists (2019 update). Nucleic Acids Res. 47, W191-W198. https://doi.org/10.1093/nar/gkz369 (2019).

59. Baryshnikova, A. Systematic functional annotation and visualization of biological networks. Cell Syst. 2, 412-421. https://doi.org/ 10.1016/j.cels.2016.04.014 (2016).

60. Kryuchkova-Mostacci, N. \& Robinson-Rechavi, M. A benchmark of gene expression tissue-specificity metrics. Brief Bioinform. 18, 205-214. https://doi.org/10.1093/bib/bbw008 (2017).

61. Vivian, J. et al. Toil enables reproducible, open source, big biomedical data analyses. Nat. Biotechnol. 35, 314-316. https://doi.org/ $10.1038 /$ nbt.3772 (2017) 
62. Mason, S. J. \& Graham, N. E. Areas beneath the relative operating characteristics (ROC) and relative operating levels (ROL) curves: Statistical significance and interpretation. Q. J. R. Meteorol. Soc. 128, 2145-2166. https://doi.org/10.1256/003590002320603584 (2002).

\title{
Acknowledgements
}

We thank members of the Lab for Cell Dynamics for helpful discussions. We are grateful to Jose Reyes, Hannah Katrina Co, and Jun-Yi Leu for their comments and suggestions on the manuscript, and Ann Mikaela Lynne Ong Co, Su-Ping Lee, the Imaging Core at the Institute of Molecular Biology, and the RNAi Core at Academia Sinica for their technical support. This work was supported by Academia Sinica Career Development Award grant (AS-CDA-108-L01); Ministry of Science and Technology, R.O.C. Grant (108-2628-B-001-002); and Undergraduate research program of the Institute of Molecular Biology, Academia Sinica, and the undergraduate research program of Ministry of Science and Technology, R.O.C. Grant (108-2813-C-001 -029 -B) to H.-K. L.

\section{Author contributions}

H.-K.L., J.-H.C., and S.-h.C. conceived the project. H.-K.L., J.-H.C., C.-C.W., and S.-h.C. designed and conducted the computational and statistical analyses. H.-K.L., J.-H.C., C.-C.W., and S.-h.C. wrote the manuscript. F.-S.H., C.D. and S.-h.C. designed the experiments, and F.-S.H., and C.D. conducted the experiments.

\section{Competing interests}

J.-H.C. is an employee of ACT Genomics. The company has no role in any part of the study. H.-K.L., J.-H.C., C.C.W, and S.-h.C. are listed as inventors on a pending patent application related to the development of C-score. The other authors declare no competing interest.

\section{Additional information}

Supplementary Information The online version contains supplementary material available at https://doi.org/ 10.1038/s41598-022-06813-4.

Correspondence and requests for materials should be addressed to S.C.

Reprints and permissions information is available at www.nature.com/reprints.

Publisher's note Springer Nature remains neutral with regard to jurisdictional claims in published maps and institutional affiliations.

\begin{abstract}
Open Access This article is licensed under a Creative Commons Attribution 4.0 International License, which permits use, sharing, adaptation, distribution and reproduction in any medium or format, as long as you give appropriate credit to the original author(s) and the source, provide a link to the Creative Commons licence, and indicate if changes were made. The images or other third party material in this article are included in the article's Creative Commons licence, unless indicated otherwise in a credit line to the material. If material is not included in the article's Creative Commons licence and your intended use is not permitted by statutory regulation or exceeds the permitted use, you will need to obtain permission directly from the copyright holder. To view a copy of this licence, visit http://creativecommons.org/licenses/by/4.0/.
\end{abstract}

(C) The Author(s) 2022 